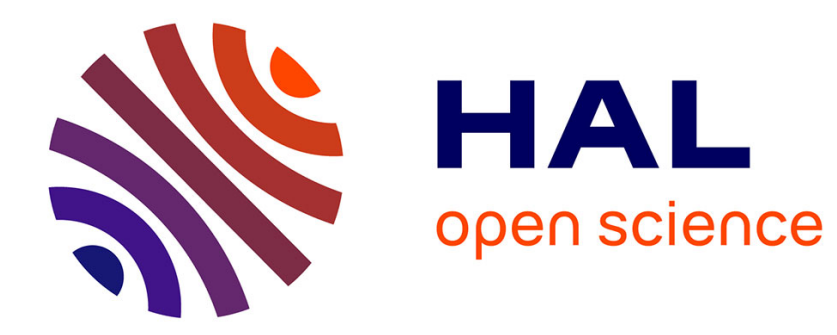

\title{
COMPOSITIONS CORPORELLES ET ACTES DE NAISSANCE DANS LE TRANSFERT
}

\author{
Sylvie Le Poulichet
}

\section{To cite this version:}

Sylvie Le Poulichet. COMPOSITIONS CORPORELLES ET ACTES DE NAISSANCE DANS LE TRANSFERT. Cliniques méditerranéennes, 2015. hal-01431678

\section{HAL Id: hal-01431678 \\ https://hal.science/hal-01431678}

Submitted on 11 Jan 2017

HAL is a multi-disciplinary open access archive for the deposit and dissemination of scientific research documents, whether they are published or not. The documents may come from teaching and research institutions in France or abroad, or from public or private research centers.
L'archive ouverte pluridisciplinaire HAL, est destinée au dépôt et à la diffusion de documents scientifiques de niveau recherche, publiés ou non, émanant des établissements d'enseignement et de recherche français ou étrangers, des laboratoires publics ou privés. 
COMPOSITIONS CORPORELLES ET ACTES DE NAISSANCE DANS LE TRANSFERT

Paru dans la revue Cliniques Méditerranéennes 91, 2015, p.41-56.

\section{LE POULICHET Sylvie}

Professeur de Psychopathologie et directrice de recherche à l'Université Paris 7- Denis Diderot .

Psychanalyste

lepoulichet.sylvie@neuf.fr

4 rue Dareau 75014 PARIS

0175572037 
Il arrive que des corps fantastiques soient mis en scène par des patients au cours de cures psychanalytiques. Ces corps fantastiques sont parfois ouverts, pluriels, hétéroclites, composés de morceaux de l'environnement non humain, de morceaux de corps d'un autre et de parties du corps propre. Un défaut de raccordement des zones érogènes rend alors précaire et parcellaire l'investissement narcissique, tout autant que l'investissement des objets. Néanmoins, nous n'avons pas à faire à un véritable corps morcelé : comment saisiralors cette énigme ?

Cette problématique m'amènera à penser la fonction des phobies pour un corps appréhendé comme partiellement invisible, non fermé, non né, puis à entendre les compulsions qui fabriquent une contenance et mettent en scène du tiers. Elle m'amènera également à présenter certaines notions issues de cette clinique, telles que le fantasme de tête coupée, puis - dans le cadre de la relation transférentielle - le jeu du mortvivant, ainsi que la dimension des actes de naissance associée au miroir identifiant que représente l'analyste.

Je rappellerai tout d'abord que la précarité des limites du corps, la confrontation avec «l'ouvert » générant des angoisses phobiques, puis les thèmes de l'invisibilité du corps et de l'identification à la machine ont longtemps été considérés comme caractéristiques de la psychose, dès lors que ces phénomènes demeurent figés et nourrissent de véritables délires ${ }^{1}$. De même, l'incertitude quant au sexe associée à l'incertitude d'être humain, ainsi qu'un surinvestissement de «l'environnement non humain ${ }^{2}$ ont pu se trouver assimilés à des manifestations psychotiques, notamment par Searles qui, quelques années plus tard, reconnaîtra que l'on rencontre très souvent ces

\footnotetext{
${ }^{1}$ On peut notamment se référer à l'article de Salomon Resnik, « Les identifications du corps dans la psychose », dans Journal de la psychanalyse de l'enfant, 20, Paris, Bayard Éditions, 1997, 84-102.

${ }^{2}$ Searles, H. 1986. L'environnement non humain, Paris, Gallimard.
} 
problématiques dans le cadre de la clinique des «états-limites » ${ }^{3}$. En effet, les thèmes et les modalités des angoisses de type psychotique éclairent vivement ce qui peut vaciller chez tout sujet traversé par des processus limites ${ }^{4}$, ces derniers n'impliquant pourtant pas les certitudes et la fixité souvent présentes dans le champ des psychoses, ni une totale éclipse du Je. Cependant, ces processus peuvent témoigner du caractère fantastique de ce qui arrive à un Moi-corpsprécocement confronté à des événements traumatiques et à des «terreurs de l'informe », un Moi-corpsqui a cherché désespérément les paroles, le regard et le désir d'un autre, sans jamais y renoncer. Et une certaine littérature n'a cessé de se nourrir de ces figures fantastiques exprimant les métamorphoses du corps et de l'espace-temps.

Un corps appréhendé comme ouvert, parfois invisible ou déformable, se tiendrait-il « de l'autre côté du miroir » ?Dans le conte de Lewis Carroll portant ce titre, c'est le miroir garant de la spécularité de l'image et du langage qui ferme la chambre, le dedans, c'est-à-dire le corps $d^{\prime}$ Alice $^{6}$, lorsque cette dernière n'en traverse pas la surface. Le miroir qui ferme et forme du corps représente l'autre qui réfléchit la singularité du sujet en l'investissant, en le regardant, en le reconnaissant et donc en le séparant. Un tel miroir identifianta en effet pour caractéristique de se retourner en enveloppe autour du corps propre. Et le miroir qui se retourne en enveloppe engendre un espace/temps possédant les propriétés de ce qui peut être réfléchi. Il engage par là même de possibles mouvements d'introjection. Cette enveloppe érogène, qui n'est autre qu'un tissu de sensations, d'images, de paroles et de désirs, n'appartient pas seulement à une dimension spatiale, car elle est également sonore et temporelle, rendant possible l'appréhension d'une continuité.

\footnotetext{
${ }^{3}$ Searles, H. 1994. Mon expérience des états-limites, Paris, Gallimard.

${ }^{4}$ Le Poulichet, S. 2003. Psychanalyse de l'informe. Dépersonnalisations, addictions, traumatismes, Paris, Aubier/Flammarion.

${ }^{5}$ Lorsque les limites entre le moi et le non moi, le dedans et le dehors, n'ont pu encore suffisamment se construire et que se présentent des événements traumatiques, le moi est l'effraction qu'il subit. Il peut alors, dans la terreur, s'appréhender comme informe. (Le Poulichet, S. Psychanalyse de l'informe, chapitre I, op.cit.)

${ }^{6}$ Carroll, L. 1871. « De 1'autre côté du miroir et ce qu'Alice y trouva », dans Euvres, Éditions Robert Laffont, 1989, 135-217.
} 
Le fait d'évoquer un « autre côté du miroir » pour un sujet implique bien qu'il aie accès à la dimension du miroir, même si ce dernier peut se révéler déformant et défaillant, de sorte que sa surface mouvante est susceptible d'être traversée et qu'un sujet peut être ainsi ponctuellement éjecté du monde de laspécularité. Se trouver tantôt devant, tantôt de l'autre côté du miroir, c'est-à-dire avec un corps parfois fermé ou parfois ouvert et invisible, c'est être exposé à des métamorphoses angoissantes qui, comme on le verra, peuvent laisser planer un doute chez le sujet concernant son appartenance à « l'espèce humaine » et concernant le fait d'être véritablement né.

Dans le conte de LewisCarroll, dès qu'Alice a traverséla surface réfléchissante et identifiante du miroir, elle pénètre dans une pièce - l'envers de la pièce initiale - où des éléments de l'environnement non humain sont vivants et parfois parlants. Cependant, en cette pièce de l'envers, Alice demeure tout à fait invisible et inaudible pour eux. Malgré tous ses efforts, Alice ne peut plus être en lien avec les objets. Hors même du champ littéraire et de l'expérience onirique dans le sommeil, il arrive qu'un Moi-corpspasse régulièrement « de l'autre côté du miroir » et revienne ponctuellement dans un monde organisé par la spécularité du langage et des images. Mais comment et pourquoi s'effectuent de telles migrations, de tels passages? Que peuvent-ils nous apprendre sur le monde fantasmatique inconscient et plus particulièrement sur les processus limites qui affectent le corps et le langage ? Ces processus se définissent précisément par le fait qu'ils organisent des franchissements de limites ou de frontières entre les verbes mettant en jeu des actions du corps, entre l'humain et le non humain, entre le vivant et le non vivant, entre l'être au monde et le non né, entre la capacité d'être en lien avec la réalité et la coupure du lien à l'autre. Ils abolissent transitoirement les oppositions distinctives et font ainsi communiquer les contraires. Les processus limites ne trouveraient-ils pas leur origine dans cette aptitude qu'ont certains sujets à passer régulièrement de l'autre côté du miroir et à en revenir ? 
L' «autre côté du miroir » n'est pas le lieu du refoulé ni du forclos, il est un lieu de l'informe et une « zone d'impersonnalisation ${ }^{7}$ qui laissent émerger de secrètes figures corporelles infantiles restées en suspens, suscitant de véritables terreurs liées à des sensations de corps partiellement ouvert, invisible ou sans contours stables. Et il importe de recueillir dans la relation thérapeutique ces étranges figures corporelles qui se sont parfois agrégé des éléments de l'environnement non humain, rendant énigmatiques les circuits du désir et l'investissement narcissique. Ce que j'ai appelé le miroir identifiant ${ }^{8}$ qui œuvre au sein de la relation thérapeutique n'a-t-il pas alors le pouvoir de susciter une nouvelle activité d'imbrication de zones érogènes s'effectuant par les voies du rêve et du transfert, véritable processus créateur engageant de nouvelles compositions corporelles ? Cependant, c'est bien entre l'analysant et l'analyste que vont surgir ces nouvelles compositions corporelles dans la relation transférentielle. Et comment se trouve alors mise en jeu l'image du corps de l'analyste ? Si, en cette clinique de l'informe, l'analyste occupe le lieu de l'autre du miroir qui nomme et reconnaît en réfléchissant, de telle sorte que s'articulent dans une continuité les dimensions du langage, de l'image et du corps, ne risque-t-il pas aussi d'être transitoirement déplacé de l'autre côté du miroir?

\section{ARIELLE DES DEUX COTÉS DU MIROIR}

Afin de donner une épaisseur clinique aux hypothèses et aux questionnements qui précèdent, je présenterai d'emblée des séquences de la cure d'Arielle.

«Est-ce que j'appartiens à l'espèce humaine ?»: c'est ainsi qu'Arielle formule la question inaugurale de sa cure. Elle précise dès la première séance : «J'ai toujours voulu être ni femme ni homme, pas incarnée », puis «ma mère ne voulait pas être

\footnotetext{
${ }^{7}$ Là où les vacillements identificatoires ont laissé en suspens les repères du Je, lui-même livré à un «blanc » de son histoire. Voir Le Poulichet, S. 2010. Les chimères du corps. De la somatisation à la création, Paris, Aubier/Flammarion.

${ }^{8} \mathrm{~L}$ 'analyste occupe le lieu de l'autre du miroir qui nomme et reconnaît en réfléchissant, de telle sorte que s'articulent les dimensions du langage, de l'image et du corps. (Le Poulichet, S. 2003. Psychanalyse de l'informe, Paris, Aubier/Flammarion).
} 
enceinte et je suis née avec la tête toute bleue, le cordon autour du cou. Ma mère m'a rapporté qu'elle s'est écriée devant la sage femme : «Comme elle est moche !». Plus tard, elle m'a laissée longtemps chez ses parents ». Ce qui suivit conféra à ces événements une valeur de condamnation, même si la dimension d'un rejet primordial n'était pas venue la couper radicalement du monde.

«Qu'est-ce que le féminin et quelle est ma place ?» questionne-t-elle encore. Et elle ajoute: «Il y a toujours eu le danger d'être rejetée ou abandonnée quelque part, n'importe quoi pouvait arriver. J'ai peur de disparaître. Ma mère était une lame coupante et imprévisible. Au fond, j'ai toujours eu envie que ma mère meure, c'est elle ou moi. Très tôt, je sentais sa haine, elle s'appropriait mes réussites et disait aux autres : «c'est moi ». Elle avait toujours peur que je meure dans un accident de voiture. Alors, j'étais si heureuse, si excitée à l'idée de retourner chez mes grands-parents, que je m'écriais : «j'aime tellement ma grand-mère que je vais la manger ».Être mangée ou manger l'autre, être tuée ou tuer l'autre? Cette possible réversibilité la hantait régulièrement.

Depuis l'adolescence, Arielle était boulimique et se faisait vomir ; elle présentait également de nombreuses phobies et compulsions. De plus, ses angoisses d'exclusion, d'abandon et de disparition s'associaient dès la première séance à une terreur touchant le visage : «Depuis longtemps, lorsque je me vois devant un miroir, c'est comme si je n'avais plus de visage, je ne me reconnais pas et je me dis qu'il va falloir en finir. Cela s'arrange si je me précipite chez ma coiffeuse, très attentionnée, qui remet bien en place mes cheveux. Alors là, j'ai à nouveau un contour ».

Je lui dis que dans le salon de coiffure, elle se voit reflétée dans le miroir à côté de cette coiffeuse si bienveillante, alors que lorsqu'elle est seule, elle se voit peut-être avec les yeux de sa mère d'autrefois. «Oui, répond-elle, on ne m'a jamais dit que j'avais quelque chose en moi ni que j'étais quelqu'un. Et ma mère ne m'a pas regardée, mon père non plus ». Puis Arielle évoque le souvenir de sa grand-mère : "Pendant longtemps, elle me mettait dans son lit pour dormir ; elle ne dormait pas avec son mari. La nuit, elle mettait toujours sa main sur mon sexe. J'étais comme un instrument pour 
elle, dans une fusion. Il n'y avait pas de différence entre son corps et le mien, on ne faisait qu'un. Là, en nommant tous ces éléments, cela se dessine, je comprends que je n'ai pas trouvé ma place. J'ai toujours été perdue par rapport à la sexualité. Je ne sais pas où je commence et où je finis devant un miroir. Mais ici je sais que j'ai un socle ». $\mathrm{Au}$ rendez-vous suivant, Arielle annonce qu'après la dernière séance, elle a pleuré dans la rue en pensant : «C'est la première fois que l'on m'entend et que j'ai été en contact avec moi-même ». Puis elle ajoute : "J'ai repensé à ma grand-mère qui était très intrusive, qui s'occupait tout le temps de mon corps en me mettant des suppositoires ou en me remplissant de nourriture, elle me remplissait de partout. Je suis claustrophobe : cela doit faire partie du même ensemble. Je me rassemble ici car je comprends que tout est lié, ce ne sont pas des particules éparpillées ».

Cependant, la semaine suivante, Arielle dit qu'en sortant de la dernière séance, une angoisse l'a saisie dans la rue en voyant en pensée mon visage se transformer en celui d'une sorcière, se déformer, devenir méchant. « J'étais stupéfaite, dit-elle, puisque c'est le lieu de la confiance ». Je lui rappelle qu'elle venait de parler, vers la fin de la précédente séance, de sa grand-mère qui était à la fois très intrusive et son « plus grand objet d'amour». «Oui, reprend-elle, elle est à double face. Je pense soudain que j'utilise depuis des années du Cif pour faire le ménage : c'est un très beau blanc mais c'est un produit très toxique. Je porte des lunettes de soleil pour qu'il n'éclabousse pas mes yeux et j'ai parfois pensé que je devrais peut-être l'avaler. Suis-je folle ? ». Je questionne : «Le bon lait grand-maternel pourrait-il se retourner en toxique ?». « Oui, dit-elle, j'ai toujours vécu là-dedans. Peut-être que je porte aussi en moi cette violence ».

La question de la folie resurgit lors de la séance suivante quand Arielle rapporte que lorsqu'elle avait onze ans, son grand-père maternel est mort subitement, lui qui représentait le seul homme fiable de la famille. La même année, le père d'Arielle abandonne le domicile familial en partant avec sa dernière maîtresse et en laissant un mot à Arielle, sa fille unique : «Tu t'occuperas bien de ta mère ». Cette mère est alors devenue alcoolique pendant plusieurs années. Arielle dit avoir plongé dans un 
cauchemar, étant constamment angoissée dans un monde flou, dépourvu de sens : « Je ne savais plus qui j'étais ». Elle devient alors agoraphobe : «Je ne sortais plus, comme si j'allais être dissoute dehors, ne plus exister ». Le trouble disparut sept ans plus tard, lorsqu'elle accoucha d'un fils. «Enfin du masculin! » dit-elle. Le fait d'avoir porté en son corps un être pourvu d'un attribut phallique avait écarté pour un temps le risque de dissolution, et avait passagèrement rendu inutile la défense phobique.

Arielle relate ensuite un premier rêve : «J'étais dans une coquille ouverte en toile, à l'abri. Des gens peignaient l'extérieur en blanc non toxique. J'étais en sécurité dans du blanc ouvert ». Elle commente : «C'est la première fois que je suis en sécurité grâce au lieu ici. J'ai compris qu'auparavant, dans mes relations aux femmes, je me soumettais et je me laissais déposséder : je m'offrais comme un steak à dévorer. Ma position initiale a été de m'offrir comme un steak et de me désintégrer. Et puis je comprends que mon sexe est resté anesthésié, congelé, depuis que ma grand-mère mettait la main dessus. J'ai l'impression d'être de la gélatine sans forme ni contour... La gélatine ? C'est le fœetus qui n'est pas né, alors c'est peut-être moi ».

Plus tard, elle dira qu'elle ne se sent pas encore incluse dans le monde, ce qu'elle associe à sa terreur face au miroir : «Mon visage reflète du monstrueux. Je viens de là...de la mère ? Elle était du sexuel cru sans limites qui se déversait par sa bouche, par ses cris, sans lien à l'autre, sans me voir. Depuis l'enfance, je fais un rêve récurrent où je suis de dos, face à ma mère qui a une tête énorme. Je suis minuscule alors que sa tête immense dépasse et fait peur. Est-ce ainsi que je vois mon visage parfois ? Est-ce que j'ai le droit d'être là ? ».

L'interrogation concernant les vœux de mort de la mère ainsi que l'angoisse du visage non vu reflétant du monstrueux, associé au «sexuel cru» et sans lien, me paraissait éclairée d'une nouvelle manière par cette image de rêve récurrente, équivalente à ce que j'ai nommé unechimère du corps $^{9}$, étrange assemblage inconscient de plusieurs corps.

\footnotetext{
${ }^{9}$ Le Poulichet,S. 2010. Les chimères du corps. De la somatisation à la création, Paris, Aubier/Flammarion.
} 


\section{DU FANTASME DE TÊTE COUPÉE À L'IMBRICATION DE ZONES ÉROGÈNES}

Selon Geneviève Haag, "la perception de la relation, celle qui fonde le premier sentiment d'enveloppe du bébé, semble être ceci : «Il faut que quelque chose entre par les yeux au fond de la tête de l'autre et de soi en miroir et fasse empreinte, puis reparte, effectuant une sorte de boucle ». Les points de retour de ces boucles finissent par faire une première sorte de tissage autour du sujet (même sujet partiel). Ce premier sentiment d'enveloppe, qui n'est pas collé à la peau, semble être un sentiment de circularité autour, et qui n'englobe pas tout le corps : il semble y avoir d'abord une émergence du tête-à-tête, la première perception de séparation est entre les deux têtes ${ }^{10}$. Elle note également qu'avant dix ou douze mois, la tête est assez soudée au tronc, mais qu'ensuite «des angoisses primitives corporelles sont ravivées, concomitantes du processus d'individuation en cours. Beaucoup d'enfants font alors des petites manœuvres de «réendossement », rentrant la tête dans les épaules... ${ }^{11}$.

Or, la clinique témoigne précisément de l'existence d'étranges formations montrant la difficulté de rattacher la tête au reste du corps, puis la fragilité de tout «tête-à-tête », ainsi que la possible réversibilité de la séparation entre les têtes. Si l'émergence d'une tête propre peut être considérée comme un processus complexemettant en jeu la perception de la séparation, rendue possible par un sentiment d'enveloppe, un tel processus doit se poursuivre à travers l'intervention d'un tiers et la rencontre d'un regard reconnaissant. A contrario, ce que je nommerai le fantasme detête coupée peut surgir à la suite d'expériences traumatiques précoces, témoignant de la précarité d'une sensation d'enveloppe corporelle propre ainsi que de l'échec partiel d'un processus de séparation.

\footnotetext{
${ }^{10}$ Haag, G. 1994. «Aux sources de la vie. Le langage préverbal et l'émergence des représentations du corps en situation psychanalytique individuelle ou groupale avec des enfants autistes », dans Dialogue, 123, p.41.

${ }^{11}$ Haag, G. 1997. « Contribution à la compréhension des identifications en jeu dans le moi corporel », dans Journal de la psychanalyse de l'enfant, 20, Paris, Bayard Éditions, p.121.
} 
Dès les premiers entretiens de la cure d'Arielle, apparaît un fantasme de tête coupée et la sensation que sa tête n'est autre que le reflet «monstrueux » de la tête maternelle. Cependant, son rêve récurrent depuis l'enfance précise ce fantasme mettant en scène la figure d'un corps dont elle ne sait « où il commence et où il finit » :le corps de la fille serait surmonté par la tête de la mère. En ce fantasme, Arielle vue par elle-même se perçoit minuscule et de dos, superposée au corps de sa mère (de face), tandis que de cet ensemble surgit l'énorme tête maternelle «qui fait peur». Or, Arielle évoque à différentes reprises sa sensation d'avoir la tête coupée du reste du corps et elle peut parfois énoncer spontanément qu'une personne veut lui couper la tête ou bien qu'ellemême voudrait couper la tête d'un autre. Par exemple : "Ma mère veut me tuer en me coupant les vivres, elle veut mettre sa tête à la place de la mienne, penser à ma place. L'image qui me vient c'est: lui arracher le visage. J'éprouve une vraie terreur d'être décapitée par ma mère. J'ai toujours vécu la tête coupée du corps et je n'ai pas de sexualité sentie ». Ces associations, parmi d'autres, montrent bien que lorsqu'un processus de séparation n'a pu totalement s'accomplir, se manifeste une relation de persécution qui hante tout rapport avec autrui : notamment, la peur d'être décapitée équivaut finalement au souhait d'arracher le visage de l'autre.

Parallèlement, le fait que le corps d'Arielle soit vu de dos en son image de rêve fondamentale s'articule bien à la nécessité qu'aucun attribut sexuel n'apparaisse à la surface de son corps : cette figure du corps serait finalement formée - dans sa partie inférieure - par la jonction de deux «parties arrières du corps » (celle de sa mère et la sienne), une tellechimère du corps se trouvant articulée à une indifférenciation sexuelle et à l'absence d'investissement de la sexualité génitale.

Quels rapports ces éléments ont-ils avec les contours flous de la tête? «L'énorme tête de la mère » se trouve elle-même appréhendée comme sans contours fixes, marquée par la folie, la démesure. En retour, Arielle éprouve souvent un sentiment de non-existence et ne reconnaît plus son propre visage flou. Mais il faut préciser que ces sensations et perceptions ne sont pas permanentes : dès lors qu'Arielle se sent véritablement regardée 
ou entendue par un autre, elle perçoit aussitôt les contours de son visage bien dessinés et se sent exister.

Il faut également ajouter que le statut de la tête, dans le cadre de l'activité fantasmatique inconsciente, se trouve souvent articulé à la sexualité. Notamment, "l'énorme tête monstrueuse » de la mère est associée par Arielle à du «sexuel cru qui se déverse », comme si le sexe se trouvait là, dans cette tête munie d'une grande bouche. Avant les changements amenés par la cure, c'était bien ce qui entrait et ce qui sortait par la bouche qui représentait le sexuel, notamment à travers la boulimie et les vomissements. Le reste de son corps était considéré par elle comme sans importance et uniquement fonctionnel. Du même coup, ce qu'Arielle désigne comme sa « position initiale », à savoir « s'offrir à l'autre comme un steak », ne manque pas de logique. Ne s'agit-il pas de se faire viande fraîche pour la grande bouche maternelle et grand-maternelle, bouche d'une ogresse qui ne pourrait se nourrir que de son enfant ?

Néanmoins, la place centrale de l'oralité dans cette problématique semble entrer en contradiction avec l'appréhension de la figure d'un corps à la tête coupée. Or, une bouche n'est pas toujours située là où on le croit. Et précisément, ce n'est qu'à la suite de plusieurs mois de cure qu'Arielle précisera la description de son rêve récurrent depuis l'enfance : en fait, le fond ou le décor du rêve était l'entrée de l'immeuble où elle vivait lorsqu'elle était enfant. Entre la "grosse tête maternelle située en hauteur et le corps minuscule d'Arielle situé de dos et en bas, se trouvait placée une boîte aux lettres sans nom : «C'est une fente, dit-elle, cela fait penser à un sexe féminin, mais aussi à une grande bouche avec rien autour, pas vivante : cela peut aspirer, mais ça ne parle pas ». En cette figure du corps, la bouche/sexe détachée se trouve donc entre la tête de la mère et le reste du corps. Une telle précision éclairait rétrospectivement les éléments précédents. Mais comment penser un orifice «avec rien autour », ou encore une zone corporelle assimilable à un élément de l'environnement non humain ?

À ce moment de la cure, Arielle relate un rêve remarquable : «Je saignais dans ma bouche, le sang s'écoulait par un trou rond dans la mâchoire supérieure. Je mettais des serviettes ou des torchons pour éponger le sang ». Elle associe rapidement ces éléments 
avec l'arrivée des règles, comme si le vagin et l'utérus étaient venus se loger dans la mâchoire supérieure et que cela saignait dans la bouche/sexe. Elle lie également cette image au «sexuel cru qui sortait de la bouche de la mère ». À la suite de ce temps d'élaboration lié au travail onirique de mise en figure, elle pouvait enfin s'approprier une zone érogène autrefois demeurée sans bord : comme si le sexe devait être rêvé dans sa bouche afin que la zone génitale puisse sortir de son anesthésie de toujours. Le fait de composer oniriquement un corps érogène fantastique semblait alors permettre d'imbriquer puis de réinvestir différentes zones érogènes. Précédemment, l'addiction c'est-à-dire sa boulimie associée à des vomissements - constituait déjà une tentative de raccordement ou d'adoption d'une zone sexuelle orale isolée, «non vivante », commune à plusieurs corps. Enfin, l'analyse suscitait maintenant, en un tel passage, un véritable travail de raccordement et de recomposition de zones érogènes vivantes.

\section{FONCTIONS DES PHOBIES POUR UN CORPS INVISIBLE, NON FERMÉ, NON NÉ}

La terreur de n'être pas véritablement sortie de la mère, relayée par l'angoisse de se dissoudre dans l'espace et d'être invisible, serait souvent mise en jeu à travers les processus limites. Cette terreur s'associe souvent à la sensation de n'être pas tout à fait formée ni fermée et elle annule partiellement l'appréhension d'une temporalité. Par des processus de déplacement et de mise en figures, certaines phobies peuvent alors organiser ultérieurement des défenses spécifiques. Plus précisément,l'agoraphobie et la claustrophobie peuvent communiquer avec des angoisses précoces de corps ouvert, sans contours propres, mal identifié. Le fait de pouvoir projeter ou déplacer dans l'espace ces éprouvés corporels permet ainsi d'user de stratégies d'évitement et de délimiter le danger.

La cure d'Arielle éclaire encore ces processus. Avant son rêve de « la bouche/utérus », elle a longtemps répété que les autres «l'envahissaient» et que cela la faisait « disparaître » car elle était « poreuse ». Sa phobie des espaces publics fermés était alors 
associée à l'angoisse que sa chair soit mélangée à celle des autres. Arielle dit : «Après la dernière séance, le métro s'est arrêté dans le tunnel, j'étais paniquée puis j'ai pensé soudain qu'un métro n'était pas un utérus. J'étais soulagée ». Elle ajoute : «Quand mon fils est né, là j'ai vu que l'on pouvait en sortir ! Donc moi aussi j'ai la possibilité d'aller à l'extérieur. C'est lorsque mon fils est né que je n'ai plus été agoraphobe. Avant, je ne savais plus qui j'étais si je sortais seule. (Silence). Le matin, je ne suis pas encore bien fermée, formée ; cela tient mieux l'après-midi, par exemple pour aller dans le métro ». Si l'on ne pouvait « sortir » de là, d'un utérus, n'était-ce pas également dans la mesure où cette sortie était restée en quelque sorte obturée par la main de la grand-mère, grande prêtresse de la non-séparation ?

À propos du non fermé et du non formé, Arielle énonce lors d'une séance suivante qu'elle a éprouvé une sensation intolérable d'intrusion, car un proche était présent dans la même pièce qu'elle lorsqu'elle préparait sa valise : «le problème, c'est que je suis ouverte » précise-t-elle. Je questionne : «Comme la valise à ce moment-là ? ». Elle acquiesce vivement puis associe : "Lorsque je ne reconnais plus mon visage, c'est comme s'il y avait un trou et que je pouvais tomber dedans. J'ai toujours eu un masque sur le visage, qui ne doit jamais changer. Dès que je pouvais être moi-même, ma grandmère n'était pas d'accord, elle ne voulait pas que je grandisse et mon visage ne devait jamais changer, pour l'éternité. Puis je ne veux pas que mon corps soit à découvert. Le corps complètement habillé, ça va, j'ai mon costume ».

Ces pensées concernant le masque et le costume, qui doivent en permanence la recouvrir, communiquent avec son angoisse d'être invisible, associéeau fait qu'elle ne s'est jamais sentie « regardée » par ses parents. De plus, ces pensées commandent un interdit de vieillir et d'être inscrite dans une trame temporelle, dans une finitude. L'invisibilité s'articule avec l'atemporalité lorsqu'un corps est resté trop longtemps « ouvert ». Si le corps risque de se dissoudre dans l'espace, il subit aussi bien souvent une éclipse temporelle. Si l'on ne doit pas naître et que l'on ne peut penser sa propre origine, on ne saurait envisager de vieillir ni de mourir. 


\section{LES COMPULSIONS QUI FABRIQUENT UNE CONTENANCE ET METTENT EN SCÈNE DU TIERS}

Une compulsion d'Arielle m'a semblé directement reliée à son étrange image du corps et à une tentative pour garantir une contenance : elle accumule dans son réfrigérateur de nombreuses boîtes de beurre « Président » à moitié consommées et ne peut les jeter que lorsqu'elles sont « périmées ». Sa mère, précise Arielle, interdisait le beurre, tandis que sa grand-mère l'en gavait. «On peut se faire une enveloppe de beurre » ajoute-t-elle. Elle mentionne cette compulsion lors d'une séance où elle est saisie par une angoisse d'abandon et par une sensation de « tomber dans le vide » car je vais bientôt partir en vacances. Cela se passe au mois de juillet, ce mois qui lui rappelle toujours le premier abandon par la mère, lorsqu'elle avait deux mois et demi. J'entends alors pour la première fois : deux «moi » et demi. Et je pense à ces boîtes de beurre comme à des contenants précieux (arrimés au signifiant «Président») renfermant une mère partie et une grand-mère trop présente (en somme : deux figures du moi), le «demi » de l'une ou de l'autre représentant plus particulièrement Arielle. Ces multiples boîtes « Président » sont mises au frais afin d'être consommées à demi, avant la date de péremption. Comme si Arielle conjurait aussi, à travers cette compulsion, sa propre date de péremption, sa propre disparition toujours redoutée. Et puis, ces boîtes bien fermées - en lesquelles une moitié du contenu subsistait - représentaient finalement son propre corps dont elle devait compulsivement mettre des exemplaires au frais. Une telle contrainte d'autoconservation, mettant en jeu un processus créateur de substituts du corps, n'était cependant pas suffisante.

Tout se passait comme si chaque matin, Arielle devait tenter de reformer, refermer son corps et lui conférer une visibilité acceptable grâce au masque et au costume. Et ceci donnait lieu à une seconde compulsion. Arielle rapporte en effet que tous les matins, il lui faut aussi faire le tour de son appartement en comptant les objets d'une manière précise pour que tout soit en place dans chaque pièce : «Je compte $2+1$ à chaque fois, ça doit faire le compte. Ce sont comme des tissus à coudre avec un fil de chair, c'est 
peut-être après cela que j'ai une peau ». Je lui dis à ce moment que le fait de relier les deux «tissus » des parents lui permet de se faire «troisième », avec une peau de « + une ». À la séance suivante, elle associe : «Ici je parle, je m'entends parler et ça se tisse, je couds en parlant. Il y a eu une greffe de peau, un tissu de chair construit en parlant. Maintenant je sens un dedans, puis un espace entre les autres et moi ». Cette fabrique d'un dedans et d'un écart était bien passée par la couture de la parole et Arielle pouvait désormais « compter trois » dans le transfert : elle, elle qui s'entend et l'analyste entre les deux.

Quant à l'élaboration d'une enveloppe corporelle, elle ne dépend pas uniquement des premières relations au maternel, comme on a coutume de le répéter en de nombreux écrits analytiques. Il faut «compter trois » pour avoir une enveloppe, sortir de la pure dualité et de la réversibilité grâce à l'introduction d'un tiers qui réfléchit un volume corporel.

Auparavant, Arielle avait pu parfois penser que l'analyste n'était peut-être personne et n'existait pas. Soudain et sans me prévenir, elle ressortait de la «bouche du métro » afin de ne pas venir à sa séance. Elle attendait que je l'appelle, que je laisse un message, pendant qu'elle faisait la morte et qu'elle pensait ma mort. Elle nous rendait donc invisibles, avant qu'elle ne resurgisse au rendez-vous suivant en demandant si j'acceptais encore de la recevoir et si le lien n'était pas cassé. Par cette mise en acte, elle avait pu ainsi jouer dans le transfert le rejet et la sortie de la bouche maternelle. De plus, elle nous avait fait disparaître et réapparaitre toutes les deux, non pas seulement pour jouer un fort- $d a^{12}$, mais surtout une alternance mort-vivant. Au jeu du mort-vivant, il faut que l'analyste réponde immédiatement : «présent ». Ce jeu du mort-vivant est bien souvent sous-tendu par une relation persécutive très précocement inscrite. Et dans le transfert peut se jouer le passage d'un «c'est elle ou moi qui sera morte » à un «c'est elle et moi qui seront vivantes ».

\footnotetext{
${ }^{12}$ Freud, S. 1920. « Au-delà du principe de plaisir », dans Essais de psychanalyse, Paris, Payot, 1981, 43-115.
} 


\section{ACTES DE NAISSANCE : ARRIVER LÀ OÙ ÇA COMMENCE}

Ce que je nommerai ici acte denaissance dans le transfert représente ce qui s'inscrit comme trace d'acte fondateur et d'origine, par les voies de la répétition et de la perlaboration dans le transfert. Arielle énonce : «L'analyse est une métamorphose pour sortir d'un régime totalitaire. Je suis devenue amie avec le monde réel, je n'ai plus peur que vous deveniez un monstre, ni de casser le lien avec vous en vous attaquant ou en vous abandonnant. J'étais décrochable du monde, non vue et sans présence, comme un lieu mort, une lettre morte ». N'était-elle pas en effet venue au monde comme une lettre morte sortie de la bouche/sexe/boîte aux lettres de la mère ?

Depuis son enfance jusqu'à ces séances, lorsque l'angoisse l'envahissait, elle avait pu partiellement et passagèrement se réfugier dans un morceau $d^{\prime}$ '«environnement non humain » susceptible de devenir un corps de substitution :par exemple se placer à l'intérieur d'un buisson dont elle touchait les feuillesafin de se sentir vivante, ou bien rester près d'un piano ou d'un orgue afin de devenir ce corps de résonance ainsi que la musique qu'il engendrait. De là provenait, entre autres choses, son incertitude d'appartenir à « l'espèce humaine », appartenance dont elle pouvait également douter à propos de ses parents. D'autres phénomènes lui échappaient et l'inquiétaient davantage, qu'elle résumait en cette formule : «Être pénétrée par le bruit et devenir le bruit, la machine, le non humain ».Elle précisait : «J'avais souvent eu peur de ma machine à laver qui tournait et faisait beaucoup de bruit. Je restais immobile et comme aspirée face au tambour qui tournait. Une partie de ma mère devait être comme une machine programmée qui ne me voyait pas, ne m'entendait pas». Face aux machines non désirantes qui tournent sur elles-mêmes, elle redoutait parfois d'être « aspirée » par ces machines sonores tenant lieu de corps parental et d'en devenir le bruit. En d'autres situations également, devenir le bruit était inconsciemment une manière de se raccorder à un autre lorsque le lien avec lui avait été rompu. Devenir l'objet partiel sonore, c'est alors devenir le bruit du lien qui n'a plus de lieu. L'identification inconsciente à l'objet 
sonore - en tant que reste ou fruit d'une rencontre manquée - serait parfois une tentative pour naître du lien.

Néanmoins, dans le devenir d'Arielle, depuis l'émergence d'actes de naissance dans la cure, le passage de la lettre morte à l'écriture de son histoire pouvait maintenant s'accomplir. Arielle apporte en séance un rêve étonnant : «Je me trouve dans un lieu étroit et je dois ouvrir des parois les unes après les autres. Puis j'arrive en bas, à l'air libre, dans un village au bord de la mer. Mais je ne sais pas où je suis et j'interroge une femme qui se trouve là, elle ne répond pas. Et je n'ai pas d'argent pour acheter un plan. Alors je tente de remonter, mais c'est impossible : les parois ont disparu. Je trouve un autre chemin : je dois traverser un livre, remonter physiquement les pages du livre pour me retrouver à la première page ». Elle associe son rêve à une naissance et au fait d'arriver à la première page du livre de son histoire pour pouvoir l'écrire elle-même : « arriver au moment où ça commence » précise-t-elle. Elle ajoute que c'est difficile car elle n'a plus son masque et son costume : « je suis à nu » dit-elle.

L'impossibilité de remonter dans l'utérus maternel, boîte à lettre morte, se trouve prononcée par cet acte de naissance onirique s'inscrivant dans le registre du transfert. De là, elle se met en position d'écrire son histoire, d'en être le sujet et l'auteur. Quant à l'événement et l'avènement de la présence, associés au fait « d'arriver au moment où ça commence », ils sont corrélatifs d'unenouvelle inscription du corps dans un espace/temps tenu par l'écriture d'une histoire.

\section{CONCLUSION}

La problématique du «naître », en de nombreuses cures analytiques, implique bien sûr la construction progressive d'un «fantasme de scène originaire ». Mais ce fantasme qui donne ancrage au corps dans le monde ne peut se déployer si ne s'élabore pas conjointement une forme constituante du narcissisme par la voie de l'investissement d'un volume corporel propre et vivant. Et cela n'est parfois possible qu'à travers un travail de composition corporelle dans le cadre de la relation transférentielle onirique 
livrant un nouvel agencement des zones érogènes. Composer signifie agencer, mettre ensemble, créer, former un tout en assemblant des éléments : en l'occurrence, il s'agit ici de composer des figures du corps tenues par le langage et les images surgissant entre l'analysant et l'analyste. La figure - souvent définie comme représentation graphique et plastique d'un ensemble de signes - engage ici la géométrie du corps pulsionnel saisi dans les sensations, les signes, les formes, les volumes. Et la composition des figures du corps dans la cure correspond à une mise en mouvement créative qui se déploie entre l'analysant et l'analyste, remettant notamment au travail la dialectique entre partie et totalité du corps érogène.

Cependant, une telle mise en mouvement suppose en bien des cas que l'analysant mette en jeu une déformation et une décomposition de l'image du corps de l'analyste, avant de pouvoir reconnaître et recoudre des formes et des liens avec le fil de la parole. C'est notamment à partir des images et des pensées venant à l'analysant concernant le corps de l'analyste appréhendé comme informe, sans existence ou comme pouvant se retourner en figure monstrueuse, qu'une telle traversée s'effectue. Transitoirement, l'analysant emporte parfois avec lui l'image du corps de l'analyste « de l'autre côté du miroir ». Et cette mise en jeu de l'image du corps de l'analyste, déformable dans l'expérience du transfert, s'associe à la matière onirique qui surgit en transformant, de telle sorte qu'apparaissent d'anciennes et de nouvelles compositions corporelles redéployant le champ des images où s'inscrivent les traits symboliques fondateurs d'une origine et d'un à-venir. L'analyste - par ses gestes, ses intonations et parfois son rire offre aussi une matière animée que l'analysant va prélever au passage. Cette matière animée présentant le corps affecté et joué, constitue l'arrière fond sur lequel peuvent progressivement s'inscrire les mots, le sens et les liens d'une façon inédite.

Finalement, à l'issue de cette traversée, c'est l'investissement de la présence, du désir, du regard et de la voix de l'analyste qui permet que s'effectue une réflexion de la libido sur le corps propre, par un effet de miroir identifiant. De là, le corps de l'analysant pourrait ne plus être éjectable du monde et les liens aux autres pourraient tenir dans une continuité. Cependant, dans le travail analytique, un miroir identifiant ne 
se réduit aucunement à un reflet, il est lui-même la création ou la couture d'un tissu de corps par la parole, condition pour que s'élaborent de nouvelles compositions corporelles, dans le temps de la fabrique d'un dedans où il est possible de « compter trois ». À ce propos, la survenue d'actes de naissance dans le registre du transfert pourrait représenter une condition pour que s'inscrive dans le temps - et de manière durable - un véritable investissement narcissique de ce dedans appréhendé comme vivant et appartenant à l'espèce humaine.

Il pourrait sembler tout à fait paradoxal d'évoquer dans un contexte analytique la dimension de la chair du langage. Pourtant, le rêve d'Arielle livre une vérité : il faut parfois traverser physiquement un livre, un volume de lettres, afin d'atteindre la première page où il sera possible de commencer à écrire/inscrire l'histoire. Traverser corporellement les pages de l'histoire serait en certains cas une modalité du transfert dans l'analyse. Il n'y a pas ici de processus créateurs sans mise en jeu du corps : fabriques de corps par le biais de compulsions, puis actes de naissance saisissant un corps onirique, mais aussi un corps de parole qui trouve son volume à travers la présence et le désir de l'autre dans la relation transférentielle. Les poètes, eux, savent depuis longtemps qu'un langage sans chair ne laisse que des lettres mortes.

\section{Bibliographie}

CAROLL, L. 1871. «De l'autre côté du miroir et ce qu'Alice y trouva », dans CEuvres, Éditions Robert Laffont, 1989, 135-217.

FREUD, S. 1920. «Au-delà du principe de plaisir », dans Essais de psychanalyse, Paris, Payot, 1981, 43-115.

HAAG, G. 1994. «Aux sources de la vie. Le langage préverbal et l'émergence des représentations du corps en situation psychanalytique individuelle ou groupale avec des enfants autistes », dans Dialogue 123, 40-58.

HAAG, G. 1997. « Contribution à la compréhension des identifications en jeu dans le moi corporel », dansJournal de la psychanalyse de l'enfant20,104-125.

LE POULICHET, S. 2003. Psychanalyse de l'informe. Dépersonnalisations, addictions, traumatismes, Paris, Aubier/Flammarion.

LE POULICHET, S. 2010.Les chimères du corps. De la somatisation à la création, Paris, Aubier/Flammarion.

RESNIK, S. 1997. «Les identifications du corps dans la psychose », dans Journal de la psychanalyse de l'enfant 20, 84-102.

SEARLES, H. L'environnement non humain, Paris, Gallimard, 1986.

SEARLES, H. Mon expérience des états-limites, Paris, Gallimard, 1994. 


\section{Résumé}

Se trouver tantôt devant, tantôt « de l'autre côté du miroir », c'est-à-dire avec un corps parfois fermé ou parfois ouvert et invisible, c'est être exposé à des métamorphoses angoissantes qui peuvent faire douter certains sujets de leur appartenance à «l'espèce humaine ». Cet article explore de nouvelles notions, telles que le fantasme de tête coupée, l'identification inconsciente au bruit résultant d'une rupture de lien, puis dans le cadre de la relation transférentielle : le jeu du mort-vivant et les dimensions des actes de naissance ainsi que du miroir identifiant. Cette recherche interroge également les compulsions qui fabriquent une contenance et mettent en scène du tiers, ainsi que les fonctions des phobies pour un corps qui s'appréhende parfois comme invisible, non fermé ou non né. La présentation d'un cas clinique permet de penser le devenir de ces formations à travers des processus créateurs mis en jeu entre l'analysant et l'analyste.

Mots-clés

Addiction, compulsion, contenance, environnement non humain, fantasme de tête coupée, fonctionnements limites, jeu du mort-vivant, miroir.

Compositions of the body and acts of birth in transference

\section{Summary}

Facing the mirror, or through the looking glass, with on one hand a closed body and on the other, an open or invisible body, isbeingexposed to anxiousmetamorphoseswhichmaysometimesinduce in somesubjects a doubt as to theirbelonging to humankind. This article explores new notions such as the fantasy of a beheaded body, the unconscious identification to the soundresulting in the breaking of a bind, then in the context of transference: the game of the living-dead and the field of the acts of birth as well as the identifyingmirror.

This researchalso questions the compulsions thatbuild up a composure and stage a third party, as well as the functions of phobias for a body apprehendingitself as open, invisible, or unborn. The study of a clinical case will examine the developments of these formations through the creativeprocessesbetweenanalysand and analyst.

Keywords

Addiction, compulsion, composure, fantasy of beheaded body,game of the living dead,non-humanenvironment, border functioning, mirror 
